4. P. Lancaster, Bounds for latent roots in damped vibration problems, SIA.M Rev. (2) 6 (1964), 121-126.

5. W. E. Roth, The equations $A X-Y B=C$, and $A X-X B=C$ in matrices, Proc. Amer. Math. Soc. 3 (1952), 392-396.

6. A. Wintner, A comparison theorem for Sturmian oscillation numbers of linear systems of second order, Duke Math. J. 24 (1958), 515-519.

The George Washington University

\title{
ORDER ISOMORPHISMS OF CONES
}

o. S. ROTHAUS

Let $C$ be a closed convex cone of vertex $\phi$ in a real normed vector space $L$. We suppose $C$ does not contain an entire straight line, and let.$\geqq$ be the order induced on $L$ by $C$. Let $S$ be some subset of $L$. A map $\varphi: S \rightarrow S$ is said to be order preserving on $S$ if $x \geqq y$ implies $\varphi(x) \geqq \varphi(y)$. We say a bijection $\varphi: S \leftrightarrow S$ is regular on $S$ if both $\varphi$ and $\varphi^{-1}$ are order preserving on $S$. (We are not assuming $\varphi$ continuous.)

Zeeman has shown [2] that if $C$ is a right circular cone in $R^{4}$, the only regular maps of $R^{4}$ are affine, with linear part a Lorentz transformation. In this paper we show that similar conclusions can be drawn under more general circumstances.

Before proceeding, we would like to record the benefit of several useful conversations with $M$. Koecher. We also note that, at the suggestion of the referee, we have modified our Proposition 1 to include the infinite dimensional case.

As part of the setting for the sequel, we shall insist that $C$ have a compact base. This means there is a continuous linear functional $h$ on $L$ such that $h(x)>0$ for $x \in C-\phi$, and the set $P=h^{-1}(1) \cap C$, called a base of the cone, is compact. Each ray in $C$ intersects $P$ exactly once, and as is customary, we call a ray passing through an extreme (respectively exposed) point of $P$ an extreme (respectively exposed) ray of $C$.

Since $P$ is the closure of the convex hull of its extreme points, $C$ is the closure of the convex hull of its extreme rays. Furthermore, let $p$ be an exposed point of $P$. There is, by definition, a continuous linear functional $s$ on $L$ and a real number $\alpha$ such that $s(x)+\alpha \geqq 0$ for $x \in P$,

Received by the editors February 9, 1966. 
with equality only for $x=p$. Then $s+\alpha h$ is a hyperplane supporting $C$ and touching $C$ only along the ray generated by $p$.

Our principal aim is to discover when a regular map of $C$ is necessarily linear. This conclusion cannot be drawn when $C$ is a half-line, or when $C$ is a Cartesian product of cones with a half-line factor. To avoid this difficulty, we confine our attention to what we shall call nonangular cones. $C$ is called nonangular if $P$ has no isolated extreme points.

Proposition 1. Let $\varphi$ be a bicontinuous regular map on $C$. Then $\varphi$ is linear on $C$.

The origin is the unique point in $C$ which is less than or equal to any point of $C$. From this it follows that $\varphi(\phi)=0$.

Let $e$ be an extreme point of $P$. Define the interval $I(a, b)$ $=\{x \in L \mid a \leqq x \leqq b\}$. Clearly $I(0, e)$ is a one dimensional closed set in $C$ consisting precisely of the points $\lambda e, 0 \leqq \lambda \leqq 1$. For if $e=x+y$ with $x, y \in C$, then $e=h(x)(x / h(x))+h(y)(y / h(y))$, so $x / h(x)=e$. The image of $I(0, e)$ is the interval $I(0, \varphi(e))$. If $\varphi(e)$ is not on an extreme ray, it is easy to see that $I(0, \varphi(e))$ is at least two dimensional. Hence $\varphi(e)$ lies on an extreme ray. A slight elaboration of this argument shows that $\varphi(\lambda e), \lambda \geqq 0$, lies on the same extreme ray. Hence there is a continuous function $\rho(\lambda)$ such that $\varphi(\lambda e)=\rho(\lambda) E(e)$, where $\rho(\lambda)$ is an order preserving homeomorphism of the half-line, and $E(e)$ is an extreme point of $P$. The function $\rho(\lambda)$ depends implicitly, of course, on $e$. It is clear that this dependence, as is that of $E$, is a continuous dependence on $e$.

For $u \in C$, the mapping $\theta_{u}: C \rightarrow C$, given by $\theta_{u}(x)=\varphi(u+x)-\varphi(u)$ satisfies the same hypothesis as does $\varphi$. Hence, by our last remarks, if $e$ is a extreme point of $P$, we have

$$
\varphi(u+\lambda e)-\varphi(u)=\rho(\lambda, e, u) E(e, u),
$$

where we have explicitly designated the dependence of the order preserving homeomorphism $\rho$ and the extreme point $E$ on the choice of $e$ and $u$.

Now let $u, v \in C$, and $u \geqq v$. Then $u+\lambda e \geqq v+\lambda e$, from which we conclude that

$$
\varphi(u)+\rho(\lambda, e, u) E(e, u) \geqq \varphi(v)+\rho(\lambda, e, v) E(e, v) .
$$

Suppose $E(e, u)$ is an exposed point of $P$. Select a supporting hyperplane to $C$ at $E(e, u)$ which touches $C$ only at points $\lambda E(e, u), \lambda \geqq 0$; i.e., we pick a linear functional $s$ on $L$ such that $s(x) \geqq 0$ for $x \in C$ with equality only for $x=\lambda E(e, u)$. We obtain 


$$
s(\varphi(u)-\varphi(v)) \geqq \rho(\lambda, e, v) s(E(e, v)) .
$$

Since $\rho(\lambda, e, v)$ approaches infinity as $\lambda$ approaches infinity, we must have $s(E(e, v))=0$. Thus $E(e, u)=E(e, v)$ in this case. Since $\theta_{u}$ is bijective, and since the exposed points of $P$ are dense in the extreme points [1], we reach the same conclusion for any extreme point $e$ of $P$. For an arbitrary pair $u, v \in C$, there is a $w \in C$ greater than both; we conclude in general that $E(e, u)$ does not depend on $u$ at all, and write henceforth $E(e, u)=E(e)$.

Now let $e$ and $f$ be distinct extreme points of $C$. Note that $E(e)$ and $E(f)$ are then distinct. We have

$$
\begin{aligned}
\varphi(u+\lambda e+\eta f) & =\varphi(u+\lambda e)+\rho(\eta, f, u+\lambda e) E(f) \\
& =\varphi(u)+\rho(\lambda, e, u) E(e)+\rho(\eta, f, u+\lambda e) E(f) \\
& =\varphi(u)+\rho(\eta, f, u) E(f)+\rho(\lambda, e, u+\eta f) E(e) .
\end{aligned}
$$

On comparing the last two equalities, we obtain

$$
\rho(\lambda, e, u)=\rho(\lambda, e, u+\eta f) .
$$

Since the closure of the convex hull of the extreme rays of $C$ is all of $C$, a simple argument, based on the last equation, shows that $\rho(\lambda, e, u)$ does not depend on $u$ at all, and we write henceforth $\rho(\lambda, e, u)=\rho(\lambda, e)$.

Referring to the long chain of equalities above, put $u=v$ to obtain

$$
\varphi(\lambda e+\eta f)=\rho(\lambda, e) E(e)+\rho(\eta, f) E(f) .
$$

Also we have

$$
\varphi((\lambda+\eta) e)=\rho(\lambda+\eta, e) E(e) .
$$

Now we make essential use of the nonangularity of the cone $C$, by letting $f$ run through a sequence of extreme points of $P$ approaching $e$. We obtain

$$
\rho(\lambda+\eta, e)=\rho(\lambda, e)+\rho(\eta, e)
$$

which shows that $\rho(\lambda, e)$ is a linear function of $\lambda$.

Hence

$$
\begin{aligned}
\varphi(u+\lambda e) & =\varphi(u)+\rho(\lambda, e) E(e) \\
& =\varphi(u)+\lambda \varphi(e),
\end{aligned}
$$

and an induction plus a limiting argument show that for any $u, v \in C$ and $\lambda, \eta \geqq 0$

$$
\varphi(\lambda u+\eta v)=\lambda \varphi(u)+\eta \varphi(v)
$$


which is what we set out to prove.

We are now going to make some applications of Proposition 1 to the finite dimensional case. We suppose for the sequel that $C$ has an interior point (which automatically forces $L$ to be finite dimensional, since then $L$ is locally compact) and that $L=R^{n}$.

First we note the following minor though useful extension of the last statement. Since $C$ has an interior, every $x \in R^{n}$ may be written, though not uniquely, in the form $x=u-v$ with $u, v \in C$. Owing to the linearity of $\varphi$ on $C$, we may extend $\varphi$ unambiguously and uniquely to a linear map $\tilde{\varphi}: \boldsymbol{R}^{n} \rightarrow \boldsymbol{R}^{n}$ by setting $\tilde{\varphi}(x)=\varphi(u)-\varphi(v) . \tilde{\varphi}$ is nonsingular, since its restriction to $C$, which has an interior, has an inverse.

We note as well that the assumption that $C$ does not contain an entire straight line guarantees in this case that $C$ has a compact base.

Finally, we note that for the applications we have in mind, continuity may be dispensed with by virtue of the following.

Lemma. Let $S$ be open in $R^{n}$ and $\varphi$ a regular map on $S$. Then $\varphi$ is continuous.

Let $a_{v} \rightarrow a$. Pick a point $p \in \operatorname{Interior} C$. It is easy to find a monotone sequence of real numbers $\epsilon_{v} \searrow 0$, such that we have

$$
a-\epsilon_{v} p \leqq a_{v} \leqq a+\epsilon_{v} p
$$

implying

$$
\varphi\left(a-\epsilon_{v} p\right) \leqq \varphi\left(a_{v}\right) \leqq \varphi\left(a+\epsilon_{v} p\right) .
$$

It is easy to show that intervals are compact, thus since $\varphi\left(a_{v}\right)$ is in the interval $I(\varphi(a-\epsilon, p), \varphi(a+\epsilon, p))$ we may assume, going to a subsequence if necessary, $\varphi\left(a_{v}\right) \rightarrow b$. This yields

$$
\varphi\left(a-\epsilon_{v} p\right) \leqq b \leqq \varphi\left(a+\epsilon_{v} p\right) .
$$

Since $\varphi^{-1}$ is order preserving, we have

$$
a-\epsilon_{v} p \leqq \varphi^{-1}(b) \leqq a+\epsilon_{v} p .
$$

Going to the limit, we conclude $\varphi^{-1}(b)=a$, which proves continuity.

Proposition 2. Let $D$ be the interior of $C$ and $\varphi$ a regular map on $D$. Then $\varphi$ is linear.

By the lemma, we know that $\varphi$ is continuous. For any $u \in D$, the $\operatorname{map} \theta_{u}: C \rightarrow C$ given by $\theta_{u}(x)=\varphi(u+x)-\varphi(u)$ satisfies the hypotheses of Proposition 1. Let $\tilde{\theta}_{u}$ be the unique extension of $\theta_{u}$ to a linear map of $R^{n}$ to $R^{n}$. We may write: 


$$
\varphi(x)=\tilde{\theta}_{u}(x-u)+\varphi(u)
$$

valid for $x \geqq u$, so $\varphi(x)$ is uniquely an affine map for $x \geqq u$. Since the affine maps so obtained for different choices of $u$ agree in pairs on open sets in $D$, they are all restrictions of a single affine map. Hence $\varphi$ itself is an affine map, with range restricted to $D$. We write

$$
\varphi(x)=L(x)+b,
$$

with $L(x)$ linear. If we let $x$ monotonely approach zero through a sequence of points in $D$, we must obtain as limit of any subsequence of $\varphi(x)$ a point in $C$ less than or equal to any point in $D$, hence less than or equal to any point in $C$. Thus $b=0$, completing the proof.

Proposition 3. Let $\varphi$ be regular on $R^{n}$. Then $\varphi$ is affine.

The proof is the same as that of Proposition 2. In addition, it is easy to see that the linear part of $\varphi$ gives a bijection of $C$.

\section{REFERENCES}

1. V. Klee, Extremal structure of convex sets, Arch. Math. 8 (1957), 234-240.

2. E. C. Zeeman, Causality implies the Lorentz group, J. Math. and Phys. (4) 5 (1964), 490-493.

Institute for Defense Analyses and

YALE UNIVERSITY 